\title{
Renal Association Clinical Practice Guideline on Vascular Access for Haemodialysis
}

\author{
Dr Richard Fluck ${ }^{a}$ and Dr Mick Kumwenda ${ }^{b}$ \\ ${ }^{a}$ Consultant Nephrologist, Derby Hospitals NHS Foundation Trust \\ ${ }^{\mathrm{b}}$ Consultant Nephrologist, Betsi Cadwaladr University Health Board, Glan Glwyd, Rhyl
}

\section{Key Words}

vascular access - arteriovenous fistula $\cdot$ arteriovenous graft · central venous catheter $\cdot$ bacteraemia $\cdot$ catheter lock solutions

\section{Introduction}

Vascular access remains a key component of haemodialysis. The ideal vascular access should provide safe and effective therapy by enabling the removal and return of blood via an extracorporeal circuit. Vascular access should be easy to use, reliable and have minimal risk to the individual receiving haemodialysis. However, the provision of good quality access, whilst it is a fundamental aspect of the treatment of haemodialysis patients, remains difficult to achieve.

Native access, in particular arteriovenous fistulae, requires prior planning yet has a high primary failure rate. Arteriovenous grafts utilising replacement of synthetic or biological material in conjunction with native vessels again require planning and surgical expertise yet have a high demand to maintain them and a high rate

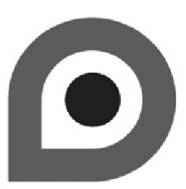

NHS Evidence Accreditation Mark of complications. Venous catheters (both tunnelled and non-tunnelled) are in common usage both as temporary access and in a smaller number of patients as the only form of access that is available, yet offer inferior therapy. Vascular access via central venous catheters provides poorer solute clearance related to the limited achieved blood flow and also a higher rate of complications.

This guideline updates the section on vascular access in the haemodialysis module of the 4th edition of the RA guidelines published on-line at www.renal.org in 2007. These guideline recommendations are based on a literature review from relevant publications in journals cited on MEDLINE, PubMed and UpToDate up to 1st May 2010. The modified GRADE system has been adopted by the Renal Association Clinical Practice Guidelines Committee and has been used to grade the recommendations in all of the modules in the 5th edition of the Renal Association guidelines. It explicitly describes both the strength of the recommendations and the quality of the underlying evidence, with the aim of maximising applicability to standard clinical practice [1-4]. The modified GRADE system grades level of expert recommendation as 'strong' (Grade 1) or 'weak'

\section{KARGER}

Fax +41613061234 E-Mail karger@karger.ch www.karger.com
Dr Richard Fluck and Dr Mick Kumwenda

Email: richard.fluck@nhs.net or mick.kumwenda@wales.nhs.uk 
(Grade 2) according to balance of benefits, risk, burden and cost. The quality or level of evidence is assessed as 'high' (Grade A), 'moderate' (Grade B), 'low' (Grade
C) or 'very low' (D) depending on factors such as study design, directness of evidence and consistency of results [1-4].

\section{References}

1 Atkins D, Best D, Briss PA, et al. Grading quality of evidence and strength of recommendations. BMJ 2004;328:1490

2 Jaeschke R, Guyatt GH, Dellinger P, et al. Use of GRADE grid to reach decisions on clinical practice guidelines when consensus is elusive. BMJ 2008;337:327-330

- 3 Uhlig K, MacLeod A, Craig J, et al. Grading evidence and recommendations for clinical practice guidelines in nephrology. A position statement from Kidney Disease: Improving Global Outcomes (KDIGO). Kidney Int 2006;70:2058-2065

4 Kidney Disease: Improving Global Outcomes. KDIGO clinical practice guidelines for the prevention, diagnosis, evaluation and treatment of Hepatitis C in chronic kidney disease. Kidney Int 2008;73(S109):S1-S99 


\section{Summary of Clinical Practice Guideline on Vascular Access for Haemodialysis}

\section{Preferred type of vascular access (Guidelines 1.1-1.3)}

\section{Guideline 1.1 - Incident patient vascular access}

We recommend that any individual who commences haemodialysis should do so with an arteriovenous fistula as first choice, an arteriovenous graft as second choice, a tunnelled venous catheter as third choice and a non tunnelled catheter as an option of necessity. (1B)

\section{Guideline 1.2 - Prevalent patient vascular access}

We suggest that any patients on long term haemodialysis should have vascular access monitored and maintained to minimise failure to allow timely planning for subsequent replacement of optimal vascular (or PD) access and avoid the need for emergency access. (2B)

\section{Guideline 1.3 - Complications related to vascular access}

We recommend that any patients on long term haemodialysis should have the risk of complications, especially infection, related to vascular access minimised by appropriate interventions. (1B)

2. Preservation of sites for native vascular access (Guidelines 2.1-2.2)

Guideline 2.1 - Preservation of peripheral veins for vascular access

We suggest that all patients that may require renal replacement therapy should have education on forearm vein preservation. (2D)

Guideline 2.2 - Preservation of peripheral veins for vascular access

We suggest that healthcare workers should avoid unnecessary venepunctures and peripheral venous access in the upper limb intended for creation of vascular access. (2C)

\section{Timing of creation of vascular access (Guidelines} 3.1-3.2)

\section{Guideline 3.1 - Planning of vascular access}

We suggest that planning for access should commence when patients enter CKD stage 4 . (2C)
Guideline 3.2 - Creation of vascular access

We recommend that the exact timing of placement of vascular access will be determined by rate of decline of renal function, co-morbidities and by the surgical pathway. (1C)

\section{Maintenance of vascular access (Guidelines} 4.1-4.4)

\section{Guideline 4.1 - Pharmacological treatment}

We suggest that there are no proven long term pharmacological interventions that have shown to improve access survival. (2C)

\section{Guideline 4.2 - Needling technique}

We suggest that buttonhole is the preferred needling technique. (2B)

\section{Guideline 4.3 - Vascular access surveillance}

We suggest that systematic observation and advanced surveillance should be employed to predict and prevent access failure. (2C)

\section{Guideline 4.4 - Intervention for failing vascular} access

We recommend that a local standard operating policy for intervention should be developed. (1C)

\section{Prevention of catheter related infections} (Guidelines 5.1-5.4)

\section{Guideline 5.1 - Minimise the use of venous catheters}

We recommend that venous catheters should be employed as a method of last resort for longer term vascular access to reduce the overall risk of infectious complications in haemodialysis patients. (1B)

\section{Guideline 5.2 - Minimising the risk of catheter related infection}

We suggest that aseptic technique should be mandatory at every manipulation of central venous dialysis catheters. (2C)

\section{Guideline 5.3 - Minimising the risk of catheter related infection}

We recommend that the catheter exit site should be cleaned with Chlorhexidine 2\%. (1B) 
Guideline 5.4-Minimising the risk of catheter related infection

We suggest that an antimicrobial or antibiotic lock solution be used to reduce catheter related bacteraemia and other infections. (2B)

\section{Complications of vascular access (Guidelines 6.1-6.4)}

\section{Guideline 6.1 - Treatment of access infection and} related bacteraemia

We recommend that venous catheters should be removed in all seriously ill haemodialysis patients with catheter related bacteraemia unless no alternative vascular access can be achieved. (1B)

\section{Guideline 6.2 - Prevention of arteriovenous aneurysmal formation}

We suggest that prevention of aneurysmal formation with good needling technique is appropriate and is the cornerstone for preserving arteriovenous fistulae. (2C)

\section{Guideline 6.3 - Treatment of ischemia related to arteriovenous fistulae or grafts}

We suggest that the development of peripheral ischaemia related to arteriovenous fistulae or grafts requires early review by the vascular access surgeon to allow proactive intervention to prevent the onset of gangrene or need for amputation. (2B)

Guideline 6.4 - Prevention and treatment of central venous catheter occlusion

We suggest that catheter occlusion may be prevented by the use of an antithrombotic lock solution and catheter occlusion should be managed by using thrombolytic agents (urokinase or t-PA) before catheter guidewire exchange or replacement. (2C)

\section{Summary of Audit Measures}

1. $65 \%$ of all incident haemodialysis patients should commence dialysis with an arteriovenous fistula

2. $85 \%$ of all prevalent patients on haemodialysis should receive dialysis via a functioning arteriovenous fistula

3. The annual Staphylococcus aureus bacteraemia rate in the prevalent haemodialysis population should be less than 2.5 episodes per $100 \mathrm{HD}$ patients and less than 1.0 for MRSA over 2 years

4. Proportion of all patients with urgent access related complications treated according to locally agreed protocols by the multidisciplinary team 
Rationale for clinical practice guidelines for vascular access for haemodialysis

\section{Preferred types of vascular access (Guidelines 1.1-1.3)}

\section{Guideline 1.1 - Incident patient vascular access}

We recommend that any individual who commences haemodialysis should do so with an AV fistula as first choice, an AV graft as second, a tunnelled venous catheter as third choice and a non tunnelled catheter as an option of necessity. (1B)

\section{Service audit marker}

The service should audit the proportion of patients whose first haemodialysis treatment is with an arteriovenous fistula.

Subgroup data may be useful in identifying where service improvement may be required. The proportion of individuals commencing haemodialysis may be subdivided as:

(a) new patients with established renal failure and known to the nephrology team for $>90$ days

(b) new patients with established renal failure and known to the nephrology team for $\leqslant 90$ days

(c) patients with a failed renal transplant

(d) patients transferred permanently from $\mathrm{PD}$ to haemodialysis.

Root cause analysis should be carried on any individual that commences haemodialysis with a venous catheter.

The service audit minimum standard

$65 \%$ of all patients commencing haemodialysis should commence with an AV fistula

\section{Research topics}

Optimal timing of arteriovenous fistula creation and value of venous and arterial mapping studies prior to surgery

Assisted patency strategy

\section{Guideline 1.2 - Prevalent patient vascular access}

We suggest that any patients on long term haemodialysis should have vascular access monitored and maintained to minimise failure to allow timely planning for subsequent replacement of optimal vascular (or PD) access and avoid the need for emergency access. (2B)
Service audit marker

A centre should measure the proportion of prevalent long term haemodialysis patients receiving dialysis via a fistula, an arteriovenous graft and a tunnelled or a non tunnelled line

The service audit minimum standard

$85 \%$ of all prevalent patients on haemodialysis should receive dialysis via a functioning arteriovenous fistula

Research topics

Monitoring and surveillance strategy and techniques Intervention options for failing access

Needling techniques

Fistula maturation and when to start needling

\section{Guideline 1.3 - Complications related to vascular access}

We recommend that any patients on long term haemodialysis should have the risk of complications, especially infection, related to vascular access minimised by appropriate interventions. (1B)

\section{Service audit markers}

1. Staphylococcus aureus (MRSA and MSSA) infection

Centres should audit all Staphylococcus aureus bacteraemia episodes recorded as episodes per 100 patient years or episodes per 100 catheter days or episodes per 100 AVF years

\section{Clostridium difficile toxin (CDT)}

Centres should audit all episodes of CDT and express rates as per 100 patient years

3. Other Multi drug resistant organisms

Data should be collected on all episodes of VRE and ESBL bacteraemia episodes per 100 patient years

4. Rupture of vascular access (fistula and graft)

The service audit minimum standard

The annual Staphylococcus aureus bacteraemia rate should be less than 2.5 episodes per $100 \mathrm{HD}$ patients and less than 1.0 for MRSA over 2 years

\section{Research topics}

Comparative analysis of lock solutions and thrombolytic agents for catheters

Bacteraemia rates in AVF and AVG related to needling technique 
Comparison of bactericidal/antibiotic locked venous catheter and AVF in the elderly or co-morbid patient (e.g. diabetes, vascular disease)

Strategies to salvage thrombosed AVF

\section{Rationale for Guidelines 1.1-1.3}

There are three principle forms of vascular access available for the treatment of patients with established renal disease with haemodialysis. These are arteriovenous fistulae, arteriovenous grafts using prosthetic or biological material and finally either tunnelled or non tunnelled catheters placed in a central vein.

There are no well constructed randomised control trials to demonstrate the superiority of one form of venous access over another but evidence from multiple studies suggest that arteriovenous fistulae are superior in terms of longevity, need for maintenance and carry less risk of vascular and infective complications compared to other types of vascular access. There is considerable variation within the United Kingdom and across the globe in the utilisation of different forms of vascular access. Both patient demographics and clinician preference may account for this variation.

Factors that might influence access provision and survival include obesity, diabetes mellitus and presence of arterial vascular disease [1]. Approximately 0.2 interventions per patient per year are done for arteriovenous fistulae compared to 1.0 intervention per patient per year for arteriovenous grafts [2]. Long-term primary access survival is estimated at approximately $90 \%$ of arteriovenous fistula after one year compared to $60 \%$ of arteriovenous graft [3]. Tunnelled venous catheters in a recent analysis have better flow characteristics and a reduced risk of infection than non-tunnelled catheters [4]. Therefore tunnelled catheters are preferred to non tunnelled catheters for the provision of vascular access.

\section{References}

1 Hodges TC, Filinger MF, Zwolak RM, et al. Longitudinal comparison of dialysis access methods: risk factors for failure. J Vas Surg 1997;26:1009

-2 Ifudu O, Mayer J, Mathew J, Fowler A, Friedman E. Haemodialysis dose is independent of type of surgically-created vascular access. Nephrol Dial Transplant 1998;13:2311-2316

3 Rayner HC, Pisoni RL, Gillespie BM, et al. Creation, cannulation and survival of arterio-venous fistulae - data from the Dialysis Outcomes and Practice Patterns Study (DOPPS). Kidney Int 2003;63:323-334
Thomson P, Stirling C, Traynor J, Morris S, Mactier R. A prospective observational study of catheter-related bacteraemia and thrombosis in a haemodialysis cohort: univariate and multivariate analyses of risk associations. Nephrol Dial Transplant 2010;25(5):1596-1660 


\section{Preservation of sites for native vascular access} (Guidelines 2.1-2.2)

Guideline 2.1 - Preservation of peripheral veins for vascular access

We suggest that all patients that may require renal replacement therapy should have education on forearm vein preservation. (2D)

Guideline 2.2 - Preservation of peripheral veins for vascular access

We suggest that healthcare workers should avoid unnecessary venepunctures and peripheral venous access in the upper limb intended for creation of vascular access. (2C)

Rationale for Guidelines 2.1-2.2

The general preferred rule for formation of arteriovenous creation is to start distally and move proximally in the non dominant arm. All pre-dialysis and dialysis patients and health professionals should be educated about the preservation of veins in the forearm and for pre-dialysis care should form part of the care bundle [1].

\section{Reference}

1 National Kidney Foundation-K/DOQI Clinical Practice Guidelines for vascular access 2000. Am J Kidney Dis 2000;37:1(Suppl 1)S137-S180 (www.kidney.org/professionals/kdoqi/guidelines.cfm) 


\section{Timing of creation of vascular access (Guidelines} 3.1-3.2)

\section{Guideline 3.1}

We suggest that planning for access should commence when patients enter CKD stage 4 . (2C)

\section{Guideline 3.2}

We recommend that the exact timing of placement of vascular access will be determined by rate of decline of renal function, co-morbidities and by the surgical pathway. (1C)

\section{Rationale for Guidelines 3.1-3.2}

For a pre-dialysis patient who has opted to commence haemodialysis, the principle goal is to initiate haemodialysis with a functioning arteriovenous fistula [1-2]. However, there are little data to determine when the optimal time for the placement of an arteriovenous fistula should occur. The determinants of the timing of such surgery should maximise the potential for initiating dialysis with an arteriovenous fistula whilst minimising the placement of unutilised access or access that is placed and then fails before the initiation of dialysis. The factors informing such a consideration include the potential for decline of an individual's renal function, the co-morbidities of the individual that affect the probability of primary success and secondary failure and finally, the logistical process of healthcare that provides an individual with an arteriovenous fistula.
Patients should be referred early enough so that they can undergo the process of the evaluation, investigation if required and then operation. Vascular access planning should commence at some point after an individual reaches CKD stage 4 . It would also be appropriate to state that an arteriovenous fistula should be placed as a minimum at three months prior to the commencement of haemodialysis and probably not more than one year from the expected date of dialysis. However, it is not uncommon in some individuals that the rate of progression is not predictable making the timing of access placement difficult. Further research is required to determine optimal timing for access creation.

For prosthetic grafts a maturation period of longer than two to three weeks after implantation is not required. Consequently, in individuals in whom an arteriovenous graft is deemed to be the appropriate access, placement can be delayed until a time closer to the expected date of dialysis.

Similarly, the major benefit of venous catheters is that they may be utilised immediately. It is however recommended that non tunnelled catheters are not utilised for more than seven days before being converted to tunnelled access. The internal jugular vein is the preferred placement site. The subclavian veins should be avoided unless absolutely necessary due to the high rate of venous stenosis associated with this site [3]. Damage to central venous structures may reduce the chances of successful arteriovenous fistula formation in the future [4].

\section{References}

1 Oliver MJ, Rothwell DM, Fung K, et al. Late creation of vascular access for haemodialysis and increased risk of sepsis. J Am Soc Nephrol 2004;15:1936-1942

2 Ortega T, Ortega F, Diaz-Corte C, et al. The timely construction of arteriovenous fistulae: a key to reducing morbidity and mortality and to improving cost management. Nephrol Dial Transplant 2005;20:598-603
Sands J. Vascular access: the past present and future. Blood Purif 2009;27:22-27

4 Vasquez MA. Vascular access for dialysis: recent lessons and new insights. Curr Nephrol Hypertens 2009;18:116-121 


\section{Maintenance of vascular access (Guidelines} 4.1-4.4)

\section{Guideline 4.1 - Pharmacological treatment}

We suggest that there are no proven long term pharmacological interventions that have shown to improve access survival. (2C)

\section{Guideline 4.2 - Needling technique}

We suggest that buttonhole is the preferred needling technique. (2B)

\section{Guideline 4.3 - Vascular access surveillance}

We suggest that systematic observation and advanced surveillance should be employed to predict and prevent access failure. (2C)

\section{Guideline 4.4 - Intervention for failing vascular access}

We recommend that a local standard operating policy for intervention should be developed. (1C)

\section{Rationale for Guidelines 4.1-4.4}

Once vascular access is formed, it is essential to maintain or enhance its function and prolong its lifetime. This section will deal with pharmacological interventions, needling techniques, surveillance and intervention.

\section{Rationale for Guideline 4.1: Anticoagulant or anti-} platelet strategies

There are no evidence based studies to support the use of either anticoagulants or anti- platelet strategies to reduce the risk of thrombosis in long term use of vascular access. A number of pharmacological interventions have been evaluated:

(a) warfarin

(b) formal anti-platelet therapy with aspirin, dipyridamole or clopidogrel

(c) alternative drugs such as Omega 3 fatty acids.

\subsubsection{Arteriovenous fistulae}

A Cochrane meta-analysis published on several shortterm studies from 1974 through to 2003 [1]. All of these trial data suggested beneficial short-term effects of antiplatelet drugs such ticlodipine, aspirin and clopidogrel but importantly several papers were thirty years or more old. In addition, the majority of the trials had short periods of follow up (one month or less). A more recent paper studied the effects of clopidogrel on the early failure of arteriovenous fistulas with a primary outcome again six week primary patency [2]. In nearly 900 participants fistula thrombosis rate was reduced to $12.2 \%$ in comparison with $19.5 \%$ in the placebo arm but crucially the secondary end point of suitability for dialysis did not differ between the two intervention arms.

\subsubsection{Arteriovenous grafts}

Crowther et al. in 2002 published on the use of low dose warfarin with a target INR of 1.4 to 1.9 [3]. The primary end point was graft thrombosis but the trial was terminated due to an increase in the number of major bleeding events in the treatment group. The conclusion was that warfarin was harmful in this setting. Again, a number of short-term studies suggested the benefit of anti-platelet therapy for the prevention of graft thrombosis. These included aspirin, dipyridamole, dipyridamole and aspirin in combination and fish oils [4-5].

\subsubsection{Venous catheters}

In 1990 Bern et al. suggested that low dose warfarin could prevent thrombosis in patients with central venous catheters [6]. The dose used was $1 \mathrm{mg}$ per day which did not affect the INR and an excess of bleeding complications was not reported. More recently warfarin has not been shown to be effective in this setting [7-9].

\section{Rationale for Guideline 4.2: Needling Technique}

There has been a recent focus on the type of needling technique employed to access arteriovenous fistulas. There are three broad techniques:

(a) area puncture (cannulation in a restricted area)

(b) rope ladder technique (needling is progressively moved up and down the length of the fistula)

(c) buttonhole technique (a tract is formed down which cannulas can be placed).

In a recent study cannulation of $\mathrm{AV}$ fistulae was compared using the button hole and rope ladder techniques [10]. Those in the buttonhole group had more unsuccessful cannulations compared to the rope ladder group but the former was associated with a significantly reduced risk of haematoma and aneurysm formation. Intervention with angioplasty was higher in the patients using the rope ladder technique. There was however an increased risk of infection associated with the buttonhole technique. It is therefore recommended that the buttonhole technique is the preferred method for fistula cannulation but enhanced measures for infection reduction 
should be employed. Area puncture is the least favoured technique.

\section{Rationale for Guideline 4.3: Vascular access surveillance}

The recognition of a failing access is crucial to (a) inform the need for intervention to maintain patency of access and (b) inform planning for further access surgery in a timely way.

Surveillance should be based around the pyramid of observation, dialysis adequacy and advanced monitoring techniques based on fistula blood flow (QA measurements).

Inspection can occur on every occasion the access is used. Observation can detect local swelling, infection, the presence of a haematoma, aneurysm and potentially the presence of stenosis. Palpation and auscultation can complement inspection particularly when a stenosis is suspected. These three steps (look, feel, listen) should be routine in the assessment of vascular access.

Such observation has been supplemented by measurement of urea clearance, venous pressure monitoring and more recently access flow measurements. Spergel et al. reported a lack of correlation between static intra pressure ratio and access blood flow and concluded that access blood flow measurements represented the best choice for advanced monitoring techniques [11].

The ideal method of measuring access blood flow should be readily applicable as a routine screening tool. A number of techniques have been utilised to measure access flow including duplex ultrasound. Ultrasound flow dilution (Transonic), glucose pump test and ionic dialysance also have been used to determine QA. At present, no technique has been shown to be conclusively beneficial in the management of access thrombosis related complications [12]. Moreover the ideal frequency to monitor access flow has not been determined. The technique used to measure QA should be easy to utilise in a routine clinical setting with little interoperator variability and high sensitivity and specificity.

What is known is that access flow is an accurate predictor of fistula and graft dysfunction. For arteriovenous grafts a blood flow rate less than $600 \mathrm{ml}$ per minute and for arteriovenous fistulae a blood flow rate less than $300 \mathrm{ml}$ may be an indication for investigation as these flow rates predict imminent thrombosis [13-14].

Rationale for Guideline 4.4: Diagnosis and intervention of failing vascular access

If a significant stenosis is suspected diagnosis by imaging and possible intervention should be timely and appropriate. Diagnosis can be carried out by duplex ultrasonography, angiography or magnetic resonance angiography. The latter modality will require contrast enhancement. Recent concerns about Gadolinium may limit MRA usage. The advantage of duplex ultrasonography is it is non invasive and in the hands of an experienced clinician is a good diagnostic tool. Angiography has the advantage of being a prelude to direct intervention at the same sitting. However, the choice of imaging modality should be based on local expertise and availability. Froger et al. found sensitivity, specificity and positive and negative predictive value of MRA in the detection of stenosed vessel segments of 97, 99, 96 and $99 \%$, respectively [15].

For venous outflow stenosis, percutaneous transluminal angioplasty is the first treatment choice [16]. For thrombosed grafts or fistulae local availability and skills will determine whether interventional radiology or surgery represents the best option for intervention. For fistula thrombosis any attempt to rescue the arteriovenous fistula should occur within 48 hours. Currently standard balloon versus cutting balloon angioplasty have not been compared in a prospective manner. Anecdotally a cutting balloon causes less discomfort to the individual and recurrence rates are lower.

Whilst it is to be hoped that patency can be maintained in either a fistula or graft, surveillance and diagnosis may indicate that a particular access is not salvageable. In such a situation planning for the next vascular access should take place in a timeframe to minimise the risk of dialysis via a tunnelled catheter. 


\section{References}

1 Da Silva AF, Escofet X, Rutherford PA. Medical adjuvant treatment to increase patency of arteriovenous fistulae and grafts. Cochrane Database of Systematic Reviews (2): CD002786, 2003

$\checkmark 2$ Dember LM Beck GJ, Allon M, et al. Effects of clopidogrel on early failure of arteriovenous fistulas for haemodialysis. JAMA 2008; 299(18):2164-2171

$\checkmark 3$ Crowther MA, Clase CM, Margetts P, et al. Low intensive warfarin is ineffective for the prevention of PTFE graft failure in patients on haemodialysis: a randomised control trial. J Am Soc Nephrol 2002;13:2331-2337

4 Polkinhorne K. Pharmacological approaches to prevent vascular access failure Nephrology 2008;13:S12-S16

$\checkmark 5$ Hiremath S, Holden RM, Fergusson D, Zimmerman DL. Antiplatelet medications in haemodialysis patients: A systematic review of bleeding rates. Clin J Am Soc Nephrol 2009;4(8):1347-1355

6 Bern MM, Bothe A, Bistrian B. Very low doses of warfarin can prevent thrombosis in central venous catheters. Ann Int Med 1990;112(6): 423-428

7 Mokrzycki MH, Jean-Jerome K, Rush H, Zdunek MP, Rosenberg SO. A randomised trial of minidose warfarin for the prevention of late malfunctions in tunnelled, cuffed haemodialysis catheters. Kidney Int 2001;59:1935-1942

8 Traynor JP, Walbaum D, Woo YM, Teenan P, Fox JG, Mactier RA. Lowdose warfarin fails to prolong survival of dual lumen venous dialysis catheters. Nephrol Dial Transplant 2001;16:645
9 Obiola CI, Conner AC, Lebon LF. Maintaining patency of haemodialysis catheters efficacy of aspirin over warfarin. Scand J Urol Nephrol 2003;37:172-176

10 Van Loon MN, Goorvaerts T, Kessels AGH, et al. Buttonhole needling of haemodialysis arteriovenous fistulae results in less complications and interventions compared to rope-ladder technique. Nephrol Dial Transplant 2010;25(1):225-230

11 Spergel LM, Holland JE, Fadem SZ, McAllister CJ, Peacock EJ. Static intra-pressure ratio does not correlate with access blood flow. Kidney Int 2004;66:1512-1516

12 McCauley P, Wingard RL, Shyr Y, Pettus W, Hakim RM, Ikizler TA. Vascular access blood flow monitoring reduces access morbidity and costs. Kidney Int 2001;60:1164-1172

13 May RE, Himmelfarb J, Yenicesu JM, et al. Predictive measures of vascular access thrombosis: a prospective study. Kidney Int 1997;52: 1656-1662

14 Tessitore N, Mansueto G, Bedogna V, et al. A prospective controlled trial on effect of percutaneous transluminal angioplasty on functioning arteriovenous fistulae survival. J Am Soc Nephrol 2003;14:1623-1627

15 Froger CL, Duijm LE, Liem YS, et al. Stenosis detection with MR angiography and digital subtraction angiography in dysfunctional hemodialysis access fistulas and grafts. Radiology 2005;234:284-291

16 Lay JP, Ashleigh RJ, Tranconi L, Ackrill P, Al-Khaffaf H. Result of angioplasty of brescia-cimino haemodialysis fistulae: medium-term follow-up. Clinical Radiology 1998;53:608-611 


\section{Prevention of catheter related infections} (Guidelines 5.1-5.4)

Guideline 5.1 - Minimise the use of venous catheters

We recommend that venous catheters should be employed as a method of last resort for longer term vascular access to reduce the overall risk of infectious complications in haemodialysis patients. (1B)

\section{Guideline 5.2 - Minimising the risk of catheter related infection}

We suggest that aseptic technique should be mandatory at every manipulation of central venous dialysis catheters. (2C)

\section{Guideline 5.3 - Minimising the risk of catheter related infection}

We recommend that the catheter exit site should be cleaned with Chlorhexidine 2\%. (1B)

\section{Guideline 5.4-Minimising the risk of catheter related infection}

We suggest that an antimicrobial or antibiotic lock solution be used to reduce catheter related bacteraemia and other infections. (2B)

\section{Rationale for Guidelines 5.1-5.4}

Infection remains the most important complication of vascular access. Pastan et al. found that the proportion of patients who died was higher among those who were dialysed with a non-cuffed or cuffed catheter compared to those dialysed with either a graft or a fistula [1]. It is clear that the risk of infection is lower for those patients dialysing on arteriovenous fistulae. However, the risk of infection is pertinent to any patient irrespective of the means by which haemodialysis is carried out.

\subsection{Incident and prevalent use of venous catheters}

Venous catheters should be employed as a method of last resort for the purpose of dialysis to reduce the overall risk of infectious complications in haemodialysis patients [2].

\subsection{Venous catheters and infection}

Infection is significantly reduced with tunnelled lines compared to non tunnelled lines [3-4]. Consequently tunnelled catheters should be preferred in all patients. Aseptic technique should be mandated at every catheter manipulation. In particular two health care professionals are necessary to connect a patient with a venous catheter to a haemodialysis monitor in order to maintain the aseptic nature of the procedure [5-6].

\subsection{Exit site strategies}

For venous catheters, the exit site remains a potential source of infection. The exit site should be cleaned with Chlorhexideine 2\%. This has been shown to be superior to povoiodine in a number of settings [7]. The exit site should be covered with a non occlusive secure dressing to protect the exit site between dialysis sessions. Patients should be educated on the importance of maintaining the integrity of the dressing and the importance of reporting problems with the exit site.

At each dialysis session the exit site should be inspected and evidence of inflammation recorded and appropriate intervention should take place. This may require enhanced cleaning, topical therapy and/or oral or intravenous antibiotic therapy depending on the extent of any infection. A Cochrane meta-analysis explored the benefit of a number of exit site strategies [8]. The use of polysporin ointment reduced the risk of catheter related bacteraemia (odds ratio 0.17) and reduced catheter related infections related to staphylococcus aureus. Interestingly there were discordant results in mortality related infection which was not reduced by mupirocin, polysporin or povoiodine therapy. Medihoney has also been tested; Johnson et al. showed that Medihoney when applied to tunnelled catheter exit site was comparable to $2 \%$ mupirocin in the number of catheter associated bacteraemia rates [9].

\subsection{Catheter lock solutions}

Catheter lock solutions have been increasingly studied. In broad terms they are divided into antimicrobial antibiotic and antimicrobial lock solutions. Catheter lock solutions used in clinical trials include gentamicin and heparin, taurolidine and citrate. Several meta-analyses have been performed in recent years all of which confirm the benefit of antibiotic lock solutions but do not give insights as to the optimal choice of catheter lock [1011]. The meta-analyses have suggested that antibiotic lock solutions are superior in terms of infection prevention but there are concerns around microbial resistance developing. These concerns have not been confirmed in any peer reviewed publications to date. In contrast, solutions such as taurolidine or citrate have been effective although possibly less so than antibiotic solutions but do have a range of side effects and do have cost implications. It is therefore recommended that some form of catheter lock solution is employed but randomised control trials and further data are required to determine the optimal solution. 


\section{References}

1 Pastan S, Soucie JM, McClellan WM. Vascular access and increased risk of death among haemodialysis patients. Kidney Int 2002;62(2):620-626

- 2 Schwab SJ, Harrington JT, Singh A, et al. Vascular access for hemodialysis. Kidney Int 1999;55:2078-2904

- 3 Oliver MJ, Callery SM, Thorpe KE, et al. Risk of bacteraemia from temporary hemodialysis catheters by site of insertion and duration of use: A prospective study. Kidney Int 2000;58:2543-2545

4 Lee T, Barker J, Allon M. Tunneled catheters in hemodialysis patients: reasons and subsequent outcomes. Am J Kidney Dis 2005;46:501-508

5 Wivell W, Bettmann MA, Baxter B. Outcomes and performance of the Tesio twin catheter system placed for hemodialysis access. Radiology 2001;221:697-703

6 Vanholder R, Canaud B Fluck R, et al. Diagnosis, prevention and treatment of haemodialysis catheter-related bloodstream infections (CRBSI): a position statement of European Renal Best Practice (ERBP). NDT Plus 2010;3:234-246 doi:10.1093/ndtplus/sfq041
7 Chaiyakunapruk N, Veenstra DL, Lipsky BA, Saint S. Chlorhexidine compared with povidone-iodine solution for vascular catheter-site care: a meta-analysis. Ann Intern Med 2002;136:792-801

8 McCann M, Moore ZE. Interventions for preventing infectious complications in haemodialysis patients with central venous catheters. Cochrane Database Syst Rev. 2010 Jan 20;(1):CD006894

-9 Johnson DW, Van Eps C Mudge DW. Randomised controlled trial of topical exit site application of Medihoney versus Mupirocin for the prevention of catheter-associated infections in haemodialysis patients. J Am Soc Nephrol 2005;16:1456-1462

10 Jaffer Y, Selby N, Taal M, et al. A meta-analysis of haemodialysis catheter locking solutions in the prevention of catheter related infections. Am J Kidney Dis 2008;51:233-241

11 James MT, Conley J, Tonelli M, et al. Meta-analysis: Antibiotics for prophylaxis against hemodilaysis catheter related infections. Ann Intem Med 2008;148:596-605 


\section{Complications of vascular access (Guidelines 6.1-6.4)}

\section{Guideline 6.1 - Treatment of access infection and related bacteraemia}

We recommend that venous catheters should be removed in all haemodialysis patients with catheter related bacteraemia unless no alternative vascular access can be achieved. (1B)

\section{Guideline 6.2 - Prevention of arteriovenous aneurysmal formation}

We suggest that prevention of aneurysmal formation with good needling technique is appropriate and is the cornerstone for preserving arteriovenous fistulae. (2C)

\section{Guideline 6.3 - Treatment of ischemia related to arteriovenous fistulae or grafts}

We suggest that the development of peripheral ischaemia related to arteriovenous fistulae or grafts requires early review by the vascular access surgeon to allow proactive intervention to prevent the onset of gangrene or need for amputation. (2B)

\section{Guideline 6.4 - Prevention and treatment of central venous catheter occlusion}

We suggest that catheter occlusion may be prevented by the use of an antithrombotic lock solution and catheter occlusion should be managed by using thrombolytic agents (urokinase or t-PA) before catheter guide wire exchange or replacement. (2C)

\section{Rationale for guidelines 6.1-6.4}

\subsection{Treatment of access related bacteraemia}

\subsubsection{Treatment of catheter related bacteraemia}

Catheter related bacteraemia episodes are relatively common - the incidence is reported at between 1-10/ 1000 patient days [1]. Moreover outcome can be poorer with higher rates of mortality and morbidity related to an increased incidence of metastatic infection (e.g. discitis, endocarditis). It is also the case that a bacteraemia episode related to another source (e.g. pneumonia) may be more complicated if a venous catheter is in place [2]. Several strategies have been suggested and employed when a bacteraemia is present [3]:

1. plan for treatment with systemic antibiotics for prolonged periods of time or
2. initiate treatment with systemic antibiotic and arrange guidewire exchange of existing access or

3. initiate treatment with systemic antibiotics and arrange removal of venous catheter or

4. use of antibiotic locks.

The dilemma is to maintain access for the purpose of dialysis whilst maximising cure of infection. In summary prolonged antibiotic therapy is required as a minimum for tunnelled catheters but the optimal approach for both tunnelled and non tunnelled catheters is catheter removal. This must be balanced against the potential options for immediate and future alternative access. Unfortunately, particularly in staphylococcus aureus bacteraemia, recurrence rates are high and are more so when venous catheters remain in-situ whether they have been exchanged over a guidewire or not [4].

Catheter related infection in the haemodialysis population is a serious and potentially lethal event. Consequently, catheter removal should be done as a matter of urgency if the patient is seriously ill or if there is evidence of metastatic infection [5-6].

\subsubsection{Treatment of infection for arteriovenous grafts}

Arteriovenous grafts have the second highest rate of infection in dialysis patients and infection of an arteriovenous graft has a worse prognosis than infection of an arteriovenous fistula [7]. Commonly, surgical exploration, repair and/or drainage are required together with a prolonged course of antibiotics. Failure to recognise graft infection may result in rupture of the graft.

\subsubsection{Treatment of arteriovenous fistula infections}

Infection of an arteriovenous fistula normally responds well to either oral or intravenous antibiotic therapy. Bacteraemia is common, may occur during cannulation and responds well to antibiotics. However, the presence of aneurysms, infected thrombi or localised abscess formation increases the risk of fistula rupture and surgical intervention may be required to either salvage or tie off the fistula.

\subsection{Aneurysms}

Vascular malformations of either arteriovenous fistulae or grafts are common. Prevention with good needling technique is appropriate and is the cornerstone of preserving arteriovenous fistulae. Aneurysm formation can lead to graft or fistula failure with thrombosis. It can also lead to sudden rupture of the access with potentially serious consequences. There are no good evidence based 
guidelines concerning the management of aneurysms in this setting but careful liaison with vascular radiology and surgical colleagues can develop local strategies for intervention.

6.3 Ischemia related to arteriovenous fistulae or grafts. Access induced ischaemia can be apparent from symptoms and clinical examination. The cause should be determined with appropriate imaging.

Access induced ischaemia is a serious complication that can result in amputation. Symptomatic ischaemia is observed in up to $8 \%$ of the haemodialysis population and is more common in elderly patients and those with pre-existing vascular disease such as diabetics or atherosclerosis. High flow arteriovenous fistulae can induce a steal phenomenon and access induced ischaemia is therefore more common in proximally located fistulae $[8,9]$. Patients may have a pale or cold hand with or without pain during exercise and/or haemodialysis but then can progress to ischaemic pain at rest and finally atrophic changes such as ulceration, necrosis and gangrene. For some patients this may require arteriovenous fistula or graft ligation, alternative access or a change to an alternative modality. Efforts to reduce flow such as banding and distal arterial extension can be performed and a DRIL procedure (distal revascularisation and internal ligation) has been performed in a number of studies successfully [8-11]. Other alternatives include the PAVA procedure (proximal arteriovenous anastamosis) where a graft interposition is used to move the AV anastamosis at the elbow to the axilla, the use of a tunnelled catheter or transfer to PD [12-14].

\subsection{Central venous stenosis and central venous} dysfunction

Central venous catheter dysfunction is a common event in haemodialysis. A reduction or cessation of blood flow interrupts therapy and reduces the adequacy of solute and fluid removal. Catheter dysfunction can be minimised by careful catheter insertion techniques under fluoroscopic and or ultrasonographic guidance [15-16] and an appropriate post-insertion bundle of care [17]. The most common form of catheter dysfunction is incomplete obstruction where fibrin formation or intraluminal thrombosis reduces the blood flow rates that can be achieved [18-19].

Prevention of catheter dysfunction by the use of an antithrombotic lock solution is recommended although the optimal lock solution and its concentration are unknown [20-21]. There are concerns about leakage of anticoagulant solutions into the systemic circulation [22]. This is more relevant with higher concentrations of citrate, serious adverse effects have been reported when using locking solutions containing $>30 \%$ citrate [23-24] and high concentration citrate locks are not recommended. Although much interest has been shown in prophylactic anticoagulation with warfarin several studies have failed to show benefit with warfarin [25-26].

Catheter occlusion should be managed by using thrombolytic agents (urokinase or t-PA) before catheter guide wire exchange. There is no evidence that one thrombolytic agent is superior the other [27]. 


\section{References}

$\checkmark 1$ Allon M, Radeva M, Bailey J, et al. HEMO Study Group. The spectrum of infection-related morbidity in hospitalised hemodialysis patients. Nephrol Dial Transpant 2005;20:1180-1186

2 Chen CH, Hsu WH, Chen HJ, Chen W, Shih CM, Hsia TC, Tu CY. Different bacteriology and prognosis of thoracic empyemas between patients with chronic and end-stage renal disease. Chest. 2007;132(2): 532-539

3 Allon M. Dialysis catheter-related bacteraemia: treatment and prophylaxis. Am J Kidney Dis 2004;45:779-791 (review)

4 Tanriover B, Carlton D, Saddekni S, et al. Bacteraemia associated with tunneled dialysis catheters: camparison of two treatment strategies. Kidney Int 2000;5:2151-2155

5 Marr KA, Sexton DI, Conlon PJ, Corey, et al. Catheter related bacteraemia and outcome of attempted catheter salvage in patients undergoing hemodialysis. Ann Intern Med 1997; 127:275-280

6 Allon M. Dialysis catheter-related bacteremia: treatment and prophylaxis. Am J Kidney Dis 2004;44(5):779-791

7 Kessler M, Hoen B, Mayeux D, et al. Bacteraemia in patients on chronic haemodialysis: A multicenter prospective survey. Nephron 1993;63: 93-100

8 Lazarides MK, Staramos DN, Panagopoulos GN, et al. Indications for surgical treatment of angioaccess-induced arterial 'steal'. J Am Coll Surg 1998;187:422-426

$\checkmark 9$ Lazarides MK, Staramos DN, Kopadis G, Maltezos C, Tzilalis VD, Georgiadis GS. Onset of arterial 'steal' following proximal angioaccess: immediate and delayed types. Nephrol Dial Transplant 2003;18:23872390

>10 Knox RC, Berman SS, Hughes JD, Gentile AT, Mills JL. Distal revascularisation-interval ligation: a durable and effective treatment for ischemic steal syndrome after haemodialysis access. J Vasc Surg 2002; 36:250-255

11 Diehl L, Johansen K, Watson J. Operative management of distal ischemia complicating upper extremity dialysis access. Am J Surg 2003;186:17-19

12 Sessa C, Riehl G, Porcu P, et al. Treatment of hand ischemia following angioaccess surgery using the distal revascularisation interval-ligation technique with preservation of vascular access: description of an 18Case Series. Ann Vasc Surg 2004;18:685-694

13 Gradman WS, Pozrikidis C. Analysis of options for mitigating haemodialysis access-related ischemic steal phenomena. Ann Vasc Surg 2004;18:59-65

\section{Acknowledgements and Conflicts of Interest}

Dr Mick Kumwenda acknowledges sponsorship from AstraZeneca and Merck Schering Plough to take part in multicentre studies and support from Amgen, Roche, Abbott, Ortho-Biotech and Synermed to attend educational meetings.
14 Zanow J, Kruger U, Scholz H. Proximalization of the arterial inflow: a new technique to treat access-related ischemia. J Vasc Surg 2006; 43(6):1216-1221

15 Kumwenda M. Two different techniques and outcomes for insertion of long-term tunnelled haemodialysis catheters. Nephrol Dial Transplant 1997;12:1013-1016

16 Bansal R, Agarwal SK, Tiwari SC, Dash SC. A prospective randomised study to compare ultrasound-guided with no ultrasound-guided double lumen internal jugular catheter insertion as a temporary haemodialysis access. Ren Fail 2005;27:561-564

17 Available at http://www.clean-safe-care.nhs.uk/index.php?pid=4 3 . Renal dialysis catheter care

18 National Kidney Foundation-K/DOQI Clinical Practice Guidelines for vascular access 2000. Am J Kidney Dis 2000;37:1 (Suppl 1)S137-S180 (www.kidney.org/professionals/kdoqi/guidelines.cfm)

19 Asif A, Cherla G, Merrill D, et al. Conversion of tunneled haemodialysis catheter-consigned patients to arteriovenous fistula. 2005;67:2399-2406

20 Hendrickx L, Kuypers D, Evenepoel P, Maes B, Messiaen T, Vanrenterghem Y. A comparative prospective study on the use of low concentrate citrate locks versus heparin lock in permanent dialysis catheters. Int J Artif Organs 2001;24:208-211

21 Weijmer MC, van den Dorpel MA, Van de Ven PJ, et al. Randomised clinical trial comparison of trisodium citrate $30 \%$ and heparin as catheter-locking solution in haemodialysis patients. J Am Soc Nephrol 2005;16:2769-2777

22 Sungur M, Eryuksel E, Yavas S, Bihorac A, et al. Exit of catheter lock solutions from double lumen acute haemodialysis catheters - an in vitro study. Nephrol Dial Transplant 2007;22:3533-3537

23 Mandolfo S, Borlandelli S, Elli A. Catheter lock solutions: its time for a change. J Vasc Access 2006;730:99-102

24 Power A, Duncan N, Singh SK, et al. Sodium citrate versus heparin catheter locks for cuffed central venous catheters: a single centre randomised controlled trial. Am J Kidney Dis 2009;53(6):1034-1041

25 Obiola CI, Conner AC, Lebon LF. Maintaining patency of haemodialysis catheters efficacy of aspirin over warfarin. Scand J Urol Nephrol 2003;37:172-176

26 Wilms L, Vercaigne LM. Does warfarin safely prevent clotting of haemodialysis catheters? A review of efficacy and safety. Semin Dial 2008;21:71-77

27 Kumwenda MJ, O’Donoghue D. The changing options for managing blocked catheters. British Journal of Renal Medicine 2006;11(4):2-4
Dr Richard Fluck wishes to declare sponsorship for educational meetings and honoraria from Baxter, Hospal-Gambro, Ortho-Biotech, Amgen, Roche and Abbott. No sponsorship has been related to vascular access apart from support from Baxter. 